\title{
¿La presencia de prótesis biliar afecta a la obtención de tejido mediante biopsia por ultrasonido endoscópico en pacientes con lesión sólida pancreatobiliar?
}

\author{
Raquel Palos-Cuellar, Enrique Murcio-Pérez*, Araceli Muñóz-Bautista, Gerardo Blanco-Velasco, \\ Omar M. Solórzano-Pineda y Oscar V. Hernández-Mondragón \\ Departamento de Endoscopia, Hospital de Especialidades, Centro Médico Nacional Siglo XXI, Instituto Mexicano del Seguro Social, Ciudad de \\ México, México
}

\section{Resumen}

Introducción y objetivo: La Sociedad Americana de Endoscopia Gastrointestinal sugiere que ante la sospecha de tumor pancreatobiliar se realice ultrasonido endoscópico (USE) para su adecuada estadificación y obtención de tejido. Sin embargo, no se ha determinado si la presencia de una prótesis biliar afecta a la obtención de tejido mediante biopsia por USE. Pacientes y métodos: Estudio retrospectivo, transversal, observacional y analítico de pacientes con lesiones sólidas pancreatobiliares con realización de biopsia por USE. Se comparó si hubo diferencias en la obtención de tejido en pacientes con presencia de prótesis biliar en comparación con aquellos sin prótesis biliar. Resultados: Se incluyeron 183 pacientes. El rendimiento general de la biopsia por USE para obtención de tejido para análisis histopatológico fue del 89.6\%, sin diferencias estadísticamente significativas entre los grupos (con prótesis biliar: $87.2 \%$ vs. sin prótesis biliar: $90.5 \% ; p=0.13$ ). Conclusión: La obtención de tejido mediante biopsia por USE en lesiones sólidas pancreatobiliares no se afecta por la presencia de una prótesis biliar.

Palabras clave: Biopsia con aguja fina. Endosonografía. Neoplasia páncreas. Neoplasia pancreatobiliar.

\section{Does biliary stent impacts on diagnostic tissue acquisition when Endoscopic Ultrasound Guided Fine Needle Aspiration Biopsy (EUS-FNA) is performed in patients with pancreatic and biliary masses?}

\begin{abstract}
Background and objectives: The American Society of Gastrointestinal Endoscopy recommends performing Endoscopic Ultrasound (EUS) for patients with suspicious pancreatic or biliary masses for adequate staging and tissue acquisition. However, it has not been determined if biliary stenting affects EUS guided tissue acquisition. Patients and methods: Retrospective, transversal, observational and analytic study from patients with pancreatic and biliary neoplasms who underwent EUS guided fine needle biopsy were compared for difference between those who had biliary stent versus those who did not have stent. Results: One hundred and eighty-three patients were included. General EUS guided tissue acquisition was $89.6 \%$ in patients with pancreatic and biliary neoplasm. There was no difference on tissue acquisition for patients with and without biliary stent (with stent $87.2 \%$ vs. without stent $90.5 \% p=0.13$ ). Conclusions: EUS guided tissue acquisition on pancreatic and biliary neoplasms is not affected by biliary stent.
\end{abstract}

Key words: Biopsy. Fine-needle. Endosonography. Pancreatic neoplasm. Biliary tract neoplasm.

Correspondencia:

*Enrique Murcio-Pérez

E-mail: murcio @ hotmail.com
Disponible en internet: 08-05-2020

Endoscopia. 2020;32(1):20-23 www.endoscopia-ameg.com 


\section{Introducción y objetivos}

Los tumores de la región pancreatobiliar son difíciles de diagnosticar exclusivamente por métodos de imagen. El diagnóstico tardío implica un pobre pronóstico, pues solo un $15-20 \%$ de los pacientes son candidatos a tratamiento quirúrgico al momento del diagnóstico'.

En tumores menores de $2 \mathrm{~cm}$ la sensibilidad de la topografía de abdomen es del $53 \%$, mientras que para la resonancia magnética es de $67 \%$ y el ultrasonido endoscópico (USE) 93\%². Debido a lo anterior la ASGE (American Society for Gastrointestinal Endoscopy) sugiere que ante la sospecha clínica de una tumoración pancreatobiliar se realicen estudios de imagen (tomografía axial computarizada o resonancia magnética) y si se sospecha que esta es resecable se realice USE para su adecuada estadificación, obtención de tejido y posterior tratamiento ${ }^{3,4}$.

A menudo se solicita USE después de la realización de colangiopancreatografía retrógrada endoscópica $(\mathrm{CPRE})^{5-7}$. Si bien el momento óptimo para la realización del USE no está bien definido, se ha sugerido que la presencia de prótesis biliares pueden interferir con la adecuada estadificación de los tumores de afección biliar distal debido al artefacto sónico que generan las prótesis $^{8-10}$.

Fusaroli, et al. realizaron un estudio en pacientes con cáncer pancreático con el objetivo de determinar la influencia de una prótesis biliar en la precisión diagnóstica del USE en tumores de cabeza de páncreas, encontrando un menor rendimiento cuando el USE se realizaba en pacientes con prótesis biliar $(47 \%)^{11}$. Sin embargo, no se ha determinado si la presencia de una prótesis biliar pudiera además afectar la obtención adecuada de tejido de la biopsia por aspiración con aguja fina (BAAF) obtenida por USE.

Por lo anterior, el presente trabajo se planteó como objetivo evaluar el impacto de la presencia o ausencia de prótesis biliar en la obtención de tejido mediante BAAF guiada por USE en pacientes con lesiones sólidas de la región pancreatobiliar.

\section{Material y métodos}

Se realizó un estudio retrospectivo, observacional, transversal y analítico en pacientes evaluados en el Departamento de Endoscopia del Hospital de Especialidades Centro Médico Nacional Siglo XXI en el periodo comprendido entre enero del 2018 y septiembre del 2019, que fueron enviados para evaluación por USE por sospecha de patología obstructiva de la región pancreatobiliar, identificada por algún estudio de imagen (ultrasonido percutáneo, tomografía abdominal, resonancia magnética o CPRE). Se incluyeron aquellos pacientes en los cuales el USE hubiera documentado una lesión sólida causante de la obstrucción con realización de BAAF guiada por USE. Se excluyeron los pacientes que hubieran tenido instrumentación quirúrgica de la vía biliar, así como aquellos cuyo expediente no fuera encontrado al momento de la recolección de la información.

Se evaluaron variables demográficas, clínicas, endosonográficas e histológicas comparando aquellos pacientes que tenían una prótesis biliar al momento de la BAAF guiada por USE con aquellos pacientes que no tuvieran prótesis biliar.

Utilizando la fórmula de diferencia de proporciones, considerando que se ha reportado un rendimiento diagnóstico del $85 \%$ en la BAAF por USE en ausencia de prótesis en comparación con un $47 \%$ en presencia de prótesis, se calculó una muestra de al menos 21 pacientes por grupo, considerando un valor alfa de 0.05 , beta de 0.20 y potencia del $80 \%$, con un intervalo de confianza del $95 \%$.

\section{Análisis estadístico}

Se describieron las variables utilizando medidas de tendencia central y dispersión de acuerdo con el tipo de variable. Se utilizó estadística descriptiva para encontrar diferencia entre los grupos mediante la prueba t de Student para variables cuantitativas de distribución normal y la prueba $U$ de Mann-Whitney para variables cuantitativas de libre distribución. Se aplicó la prueba de chi cuadrada para variables cualitativas. Se considero estadísticamente significativo un valor de $p<0.05$. Se utilizó el programa SPSS versión 24.

\section{Consideraciones éticas}

Este protocolo fue aprobado por el Comité de Ética de nuestro hospital con el número R-2019-3601-234. Debido a la naturaleza del estudio, se considera un estudio con riesgo menor al mínimo.

\section{Resultados}

Se incluyeron 183 pacientes. El sexo femenino representó el $59 \%(n=108)$ de la muestra. La media de edad fue de $64.8 \pm 14.2$ años. La estenosis biliar fue de localización proximal en 46 pacientes $(25.1 \%$ ) y distal en 137 pacientes (74.9\%), y se encontró presencia de prótesis biliar en 47 pacientes $(25.7 \%)$. No hubo 
Tabla 1. Características generales de los pacientes con lesión sólida pancreatobiliar y realización de BAAF por USE

\begin{tabular}{|c|c|c|c|c|}
\hline & $\begin{array}{l}\text { Todos los pacientes } \\
\qquad(\mathrm{n}=183)\end{array}$ & $\begin{array}{l}\text { Con prótesis biliar } \\
\qquad(n=47)\end{array}$ & $\begin{array}{c}\text { Sin prótesis biliar } \\
(\mathbf{n}=136)\end{array}$ & $\mathbf{p}$ \\
\hline Edad, años ( \pm SD) & $64.85 \pm 14.2$ & $66.6 \pm 12.9$ & $64.2 \pm 14.6$ & $0.13^{*}$ \\
\hline Sexo femenino, $\%$ (n) & $59 \%(108)$ & $46.8 \%(22)$ & $63.2 \%(86)$ & $0.06^{\dagger}$ \\
\hline Estenosis proximal, \% (n) & $25.1 \%(46)$ & $29.7 \%(14)$ & $23.5 \%(32)$ & $0.12^{\dagger}$ \\
\hline Estenosis distal, \% (n) & $74.9 \%(137)$ & $70.3 \%(33)$ & $76.5 \%(104)$ & $0.22^{\dagger}$ \\
\hline $\begin{array}{l}\text { Diagnóstico final } \\
\text { Adenocarcinoma de páncreas, (n) } \\
\text { Colangiocarcinoma, (n) } \\
\text { Otros (n) }\end{array}$ & $\begin{array}{l}53 \\
29 \\
22\end{array}$ & $\begin{array}{c}10 \\
7 \\
3\end{array}$ & $\begin{array}{l}43 \\
22 \\
19\end{array}$ & $0.23^{\dagger}$ \\
\hline
\end{tabular}

*Prueba t de Student.

tPrueba $\chi 2$.

BAAF: biopsia por aspiración con aguja fina; SD: desviación estándar; USE: ultrasonido endoscópico.

Tabla 2. Comparación de la obtención de tejido mediante BAAF por USE entre los grupos de acuerdo con la presencia de prótesis biliar

\begin{tabular}{|l|c|c|c|}
\hline & $\begin{array}{c}\text { Con prótesis } \\
\text { biliar (n= 47) }\end{array}$ & $\begin{array}{c}\text { Sin prótesis } \\
\text { biliar (n= 136) }\end{array}$ & p \\
\hline $\begin{array}{l}\text { Adecuada obtención } \\
\text { de tejido mediante } \\
\text { BAAF, \% }\end{array}$ & $87.2 \%$ & $90.5 \%$ & $0.13^{*}$ \\
\hline $\begin{array}{l}\text { Tejido insuficiente } \\
\text { para diagnóstico, } \\
\% \text { (n) }\end{array}$ & $12.7 \%(6)$ & $9.5 \%(13)$ & $0.33^{\dagger}$ \\
\hline $\begin{array}{l}\text { *Prueba } \chi 2 . \\
\text { 'Prueba exacta de Fisher. } \\
\text { BAAF: biopsia por aspiración con aguja fina; USE: ultrasonido endoscópico. }\end{array}$ & \\
\hline
\end{tabular}

diferencias significativas en edad, sexo o ubicación de la estenosis entre ambos grupos (Tabla 1).

El rendimiento general de la BAAF por USE para obtención de tejido para análisis histopatológico fue del $89.6 \%$, sin diferencias estadísticamente significativas entre los grupos (con prótesis biliar: $87.2 \%$ vs. sin prótesis biliar: $90.5 \% ; p=0.13$ ). Hubo diagnóstico histopatológico insuficiente en 19 pacientes, que representan el $10.4 \%$ (13 sin prótesis biliar [7.1\%] y 6 con prótesis biliar [3.2\%]; $p=0.13$ ) (Tabla 2).

El subanálisis de acuerdo con la localización de la lesión (proximal o distal) no mostró diferencias en la obtención de tejido para análisis histopatológico (Tabla 3).

\section{Discusión}

La biopsia guiada por USE es un método preciso para el diagnóstico de tumores pancreatobiliares
Tabla 3. Comparación de la obtención de tejido mediante BAAF por USE entre los grupos de acuerdo con la localización de la estenosis

\begin{tabular}{|l|c|c|c|c|}
\hline Parámetro & $\begin{array}{c}\text { Todos los } \\
\text { pacientes } \\
(\mathbf{n}=\mathbf{1 9})\end{array}$ & $\begin{array}{c}\text { Con } \\
\text { prótesis } \\
\text { biliar } \\
(\mathbf{n}=\mathbf{6})\end{array}$ & $\begin{array}{c}\text { Sin } \\
\text { prótesis } \\
\text { biliar } \\
(\mathbf{n}=\mathbf{1 3})\end{array}$ & $\mathbf{p}$ \\
\hline $\begin{array}{l}\text { Estenosis } \\
\text { proximal, \% (n) }\end{array}$ & $13 \%(6)$ & $50 \%(3)$ & $50 \%(3)$ & $0.253^{*}$ \\
\hline $\begin{array}{l}\text { Estenosis } \\
\text { distal, \% (n) }\end{array}$ & $9.5 \%(13)$ & $23 \%(3)$ & $76 \%(10)$ & $0.61^{*}$ \\
\hline
\end{tabular}

*Prueba exacta de Fisher.

BAAF: biopsia por aspiración con aguja fina; USE: ultrasonido endoscópico.

(sensibilidad $>85 \%$ ). El rendimiento diagnóstico en toda la población incluida en este estudio fue del $89.6 \%$, lo cual se considera aceptable y coincide con los resultados reportados por estudios previos ${ }^{1-2}$.

Se ha sugerido que la presencia de prótesis biliares puede interferir con la estadificación de los tumores de cabeza de páncreas ${ }^{12}$. Fusaroli, et al. estudiaron 65 pacientes subsecuentes (19 con prótesis biliar y 46 sin prótesis biliar), encontrando que el estadio quirúrgico T4 era más frecuente en pacientes con prótesis biliar (53 vs. $22 \% ; p=0.014)$. El estadiaje por USE fue correcto en el $85 \%$ de los pacientes sin prótesis biliares y en el $47 \%$ de los pacientes con prótesis, lo cual condiciona una disminución del $38 \%$ en el rendimiento diagnóstico ${ }^{11}$.

En este estudio, observamos que la presencia de prótesis biliar no influyó en la obtención de tejido cuando se realiza BAAF por USE en pacientes con obstrucción biliar (con prótesis biliar: $87.2 \%$ vs. sin prótesis 
biliar: $90.5 \% ; p=0.13$ ). Lo anterior sugiere que a pesar de que la prótesis biliar puede generar interferencia sónica, esta no afecta el desempeño de la aguja para la toma de la biopsia.

Un hallazgo relevante fue que la obtención de tejido mediante BAAF por USE no fue diferente en lesiones de localización distal en comparación con estenosis de localización distal, siendo en ambos grupos cercanos al $90 \%$. Estos hallazgos contrastan con estudios previos donde se sugiere que el rendimiento diagnóstico es mucho menor en estenosis proximales. La sensibilidad y especificidad de la BAAF por USE oscilan entre el 81 y el $91 \%$ para estenosis distales. Sin embargo, su sensibilidad para las estenosis biliares proximales disminuye, oscilando entre el 25 y el $89 \%$. Dicho hallazgo se explica debido a que solo incluimos pacientes donde se encontró lesión sólida causante de la obstrucción proximal, lo cual sugiere que, una vez localizada la lesión, la obtención de tejido mediante BAAF por USE en lesiones proximales y distales no es técnicamente diferente.

Se encontró muestra insuficiente en el $10 \%$ de los pacientes, lo cual concuerda con la literatura mundial, y esto fue independiente de la presencia o no de prótesis biliar. Estudios previos han mostrado que la toma de BAAF por USE tiene una sensibilidad general del 66 y $80 \%$ y especificidades del 100 y $80 \%$ en el diagnóstico de estenosis biliares malignas ${ }^{13,14}$.

Nuestro estudio tiene varias limitaciones, como su diseño retrospectivo y haber sido realizado en un solo centro. Además, no se pudo comparar la precisión diagnóstica de la BAAF por USE con el estándar de oro que es la pieza quirúrgica. Sin embargo, su diseño tuvo la intención de determinar si el desempeño de la BAAF por USE en la obtención de tejido se ve afectado por la presencia de una prótesis biliar.

\section{Conclusiones}

La obtención de tejido mediante BAAF por USE en lesiones sólidas pancreatobiliares no se ve afectada por la presencia de una prótesis biliar.

\section{Financiamiento}

La presente investigación no ha recibido ninguna beca específica de agencias de los sectores público, comercial, o sin ánimo de lucro.

\section{Conflicto de intereses}

Los autores declaran no tener conflicto de intereses.

\section{Responsabilidades éticas}

Protección de personas y animales. Los autores declaran que para esta investigación no se han realizado experimentos en seres humanos ni en animales.

Confidencialidad de los datos. Los autores declaran que han seguido los protocolos de su centro de trabajo sobre la publicación de datos de pacientes.

Derecho a la privacidad y consentimiento informado. Los autores han obtenido el consentimiento informado de los pacientes y/o sujetos referidos en el artículo. Este documento obra en poder del autor de correspondencia.

\section{Bibliografía}

1. Baron TH, Mallery JS, Hirota WK, Goldstein JL, Jacobson BC, Leighton JA, et al. The role of endoscopy in the evaluation and treatment of patients with pancreaticobiliary malignancy. Gastrointest Endosc. 2003;58:643-9.

2. Levy MJ, Baron TH, Clayton AC, Enders FB, Gostout CJ, Halling KC, et al. Prospective evaluation of advanced molecular markers and imaging techniques in patients with indeterminate bile duct strictures. Am J Gastroenterol. 2008;3:1250-63.

3. Kiriyama S, Kozaka K, Takada T, Strasberg SM, Pitt HA, Gabata T, et al. Tokyo Guidelines 2018: diagnostic criteria and severity grading of acute cholangitis. J Hepatobiliary Pancreat Sci. 2018;25:17-30.

4. American Society for Gastrointestinal Endoscopy (ASGE) Standards of Practice Committee, Anderson MA, Appalaneni V, Ben-Menachem T, Decker GA, Early DS, Evans JA, et al. The role of endoscopy in the evaluation and treatment of patients with biliary neoplasia. Gastrointest Endosc. 2013;77:167-74.

5. Hunt GC, Faigel DO. Assessment of EUS for diagnosing, staging, and determining resectability of pancreatic cancer: a review. Gastrointest Endosc. 2002;55:232-7.

6. Agarwal B, Abu Hamda E, Molke KL, Correa AM, Ho L. Endoscopic ultrasound-guided fine-needle aspiration and multidetector spiral CT in the diagnosis of pancreatic cancer. Am J Gastroenterol. 2004;99:844-50.

7. Bhutani MS, Hawes RH, Baron PL, Sanders-Cliette A, van Velse A, Osborne JF, et al. Endoscopic ultrasound-guided fine-needle aspiration of malignant pancreatic lesions. Endoscopy. 1997;29:854-8.

8. Chen CH, Tseng LJ, Yang CC, Yeh YH, Mo LR. The accuracy of endoscopic ultrasound, endoscopic retrograde cholangiopancreatography, computed tomography, and transabdominal ultrasound in the detection and staging of primary ampullary tumors. Hepatogastroenterology. 2001;48:1750-3

9. Nikfarjam M, Muralidharan V, McLean C, Christophi C. Local resection of ampullary adenocarcinomas of the duodenum. ANZ J Surg. 2001;7:529-3.

10. Chen $\mathrm{CH}$, Tseng LJ, Yang CC, Yeh YH. Preoperative evaluation of periampullary tumors by endoscopic sonography, transabdominal sonography, and computed tomography. J Clin Ultrasound. 2001;29:313-21.

11. Fusaroli $P$, Manta R, Fedeli $P$, Maltoni S, Grillo A, Giovannini E, et al. The influence of endoscopic biliary stents on the accuracy of endoscopic ultrasound for pancreatic head cancer staging. Endoscopy. 2007;39:813-7.

12. Cannon ME, Carpenter SL, Elta GH, Nostrant TT, Kochman ML, Ginsberg GG, et al. EUS compared with CT, magnetic resonance imaging, and angiography and the influence of biliary stenting on staging accuracy of ampullary neoplasms. Gastrointest Endosc. 1999;50:27-33.

13. El Hajj II, Al-Haddad M. Endoscopic ultrasound-guided tissue acquisition of pancreatic masses. Tech Gastrointest Endosc. 2018;20:30-8.

14. Cazacu I, Luzuriaga A, Saftoiu A, Vilmann P, Bhutani M. A quarter century of EUS-FNA: Progress, milestones, and future directions. Review article. Endosc Ultrasound. 2018;7:141-60. 